\title{
Cardiovascular Profile of Valbenazine: Analysis of Pooled Data from Three Randomized, Double-Blind, Placebo-Controlled Trials
}

\author{
Dao Thai-Cuarto ${ }^{1}$ Christopher F. O'Brien ${ }^{1} \cdot$ Roland Jimenez $^{1}$ - Grace S. Liang ${ }^{1}$ • \\ Joshua Burke ${ }^{1}$
}

Published online: 7 December 2017

(c) The Author(s) 2017. This article is an open access publication

\begin{abstract}
Introduction Valbenazine is a novel vesicular monoamine transporter 2 inhibitor approved for the treatment of tardive dyskinesia in adults.

Objective Using data from double-blind, placebo-controlled trials, analyses were conducted to evaluate the cardiovascular effects of once-daily valbenazine in patients with a psychiatric disorder who developed tardive dyskinesia after exposure to a dopamine-blocking medication.

Methods Data were pooled from three 6-week, doubleblind, placebo-controlled trials: KINECT (NCT01688037), KINECT 2 (NCT01733121), and KINECT 3 (NCT02274558). Data from the 42-week valbenazine extension period of KINECT 3 were also analyzed. Outcomes of interest included cardiovascular-related treatment-emergent adverse events, vital sign measurements, and electrocardiogram parameters.

Results The pooled safety population included 400 participants (placebo, $n=178$; valbenazine $40 \mathrm{mg} /$ day, $n=110$; valbenazine $80 \mathrm{mg} /$ day, $n=112$ ). A history of cardiac disorders was present in $11.8 \%$ of participants, and $74.3 \%$ were taking a concomitant medication with known potential for QT prolongation. Mean changes from baseline to week 6 in supine vital signs and QTcF (Fridericia correction) were as follows for placebo, valbenazine $40 \mathrm{mg} / \mathrm{day}$, and valbenazine $80 \mathrm{mg} /$ day, respectively: systolic blood pressure $(0.2,-2.1$, $-1.8 \mathrm{mmHg})$, diastolic blood pressure $(-0.1,-1.6$, $-1.2 \mathrm{mmHg})$, heart rate $(-1.7,-2.2,-1.7 \mathrm{bpm}), \mathrm{QTcF}$ interval $(1.2,1.1,2.1 \mathrm{~ms})$; all $p>0.05$ for valbenazine vs.
\end{abstract}

Dao Thai-Cuarto

dthai@neurocrine.com

1 Neurocrine Biosciences, Inc., 12780 El Camino Real, San Diego, CA 92130, USA placebo. No statistically significant differences were observed between placebo and valbenazine in cardiovascular-related, treatment-emergent adverse events. No notable additional effects on cardiovascular outcomes were found with up to 48 weeks of valbenazine treatment.

Conclusions Results from double-blind, placebo-controlled trials showed no apparent difference between valbenazine and placebo on cardiovascular outcomes. No additional cardiovascular risk was detected during a longer extension study with valbenazine.

\section{Key Points}

Data from clinical trials of valbenazine, which is approved for the treatment of tardive dyskinesia in adults, were analyzed to assess the cardiovascular profile of this medication.

Results indicated that valbenazine had minimal cardiovascular effects in patients with tardive dyskinesia who had a concurrent psychiatric disorder, the majority of whom were taking concomitant antipsychotic or antidepressant medications.

\section{Introduction}

In patients with psychiatric disorders, cardiovascular diseases account for approximately $20-30 \%$ of co-morbid medical diagnoses and $30 \%$ of deaths, higher than in the 
general population [1-5]. Prominent cardiovascular risk factors in patients with psychiatric disorders include obesity, hypertension, diabetes mellitus, atrial fibrillation, peripheral vascular disease, hyperlipidemia, and ischemic heart disease [1, 6, 7]. Direct pathophysiological connections between psychiatric illness and cardiovascular disease may exist, particularly via the sympathetic nervous system and the hypothalamic-pituitary axis, both of which are activated by increased stress, anxiety, and depression and are also involved in cardiovascular control [1, 3, 8]. Psychiatric illness and cardiovascular risk may also be indirectly linked through social or behavioral factors such as non-adherence to medical advice or treatment, social isolation, smoking, overeating, and a sedentary lifestyle $[1,3,8,9]$.

The risk of cardiovascular disease in patients with psychiatric disorders can be further complicated by the need for medications associated with adverse effects such as increased blood pressure, hypotension, cerebrovascular events, and electrocardiogram (ECG) QT prolongation $[6,7]$. Cardiovascular warnings can be found in the prescribing information for many antipsychotic medications (e.g., quetiapine, risperidone, haloperidol) and antidepressant medications (e.g., citalopram, fluoxetine) [7, 10]. Antipsychotic medications have also been associated with metabolic changes that may affect cardiovascular health, including hyperglycemia/diabetes mellitus, dyslipidemia, and weight gain [6]. Some mechanisms by which these medications may affect cardiovascular function include ion channel blockade (e.g., calcium, sodium, potassium), conduction disturbance or sinus node abnormality, ventricular dysfunction, and hypotension [2, 6]. Psychotropic medications may also impair cardiovascular function by delaying ventricular repolarization, which can result in a prolonged QT interval and possible torsades de pointes, an unpredictable arrhythmia that is difficult to manage $[2,11]$.

Valbenazine is a novel vesicular monoamine transporter 2 inhibitor approved by the US Food and Drug Administration on 11 April, 2017 for treating adults with tardive dyskinesia (TD) [12], a movement disorder associated with exposure to dopamine receptor-blocking agents such as antipsychotic medications [13-15]. Valbenazine clinical trials enrolled patients with schizophrenia, schizoaffective disorder, or a mood disorder, and stable doses of concomitant medications (e.g., antipsychotics and antidepressants) were allowed for psychiatric management [16-18]. Valbenazine was well tolerated in clinical trials, and the prescribing information for this new drug (40-80 mg/day, once-daily dosing) carries no black-box warnings or contraindications as of October 2017 [19]. The label includes only two warnings and precautions: somnolence, which can impair driving ability; and possible QT prolongation, with recommended avoidance in patients with congenital long
QT syndrome or with arrhythmias associated with a prolonged QT interval [19]. In the current report, data from three placebo-controlled, phase II/III trials and one extension trial were analyzed to provide more detailed information regarding the cardiovascular profile of valbenazine in patients with TD and schizophrenia, schizoaffective disorder, or a mood disorder.

\section{Methods}

\subsection{Trial Design and Participants}

The cardiovascular profile of once-daily valbenazine was assessed using pooled data from three multicenter, randomized, double-blind, placebo-controlled (DBPC) phase II/III trials in adults with TD: KINECT (NCT01688037) [20], KINECT 2 (NCT01733121) [16], and KINECT 3 (NCT02274558) [17]. In KINECT, participants were randomized to valbenazine $50 \mathrm{mg} /$ day (6 weeks), valbenazine $100 \mathrm{mg} /$ day ( 2 weeks) then $50 \mathrm{mg} /$ day (4 weeks), or placebo (6 weeks) followed by a 6-week, open-label phase of valbenazine $50 \mathrm{mg} /$ day. In KINECT 2, participants randomized to valbenazine received a starting dose of $25 \mathrm{mg} /$ day that was escalated incrementally by $25 \mathrm{mg} /$ day every 2 weeks to a maximum of $75 \mathrm{mg}$ /day depending on clinical response. In KINECT 3 (phase III trial), participants were randomized to valbenazine $40 \mathrm{mg} / \mathrm{day}$, valbenazine $80 \mathrm{mg} / \mathrm{day}$, or placebo for 6 weeks followed by a blinded valbenazine extension period. During the extension period, participants received up to 42 weeks of treatment with valbenazine (40 or $80 \mathrm{mg} / \mathrm{day}$, blinded dose) followed by a 4-week washout period [18].

The trials included men and women, aged 18-85 years, who had a diagnosis of schizophrenia or schizoaffective disorder, or mood disorder (KINECT 2 and KINECT 3 only), in addition to TD as per the Diagnostic and Statistical Manual of Mental Disorders, 4th Edition criteria for at least 3 months prior to screening. Participants were required to have moderate or severe $\mathrm{TD}$ at screening, based on a qualitative assessment of Abnormal Involuntary Movement Scale videos by external reviewers. Participants were also required to be psychiatrically stable, as indicated by a Brief Psychiatric Rating Scale total score $<50$.

Exclusion criteria for the trials included: any active, clinically significant, and/or unstable medical condition; known history of long QT syndrome or cardiac tachyarrhythmia; QTcF (Fridericia correction) of $>450 \mathrm{~ms}$ for men or $>470 \mathrm{~ms}$ for women; and the presence of any clinically significant cardiac abnormality. Participants requiring a concomitant medication known to prolong the QT interval were allowed to participate based on a medical review. Only individuals with the capacity to provide 
consent (based on the University of California, San Diego Brief Assessment of Capacity to Consent) were allowed to participate in the clinical trials.

\subsection{Data Analyses}

Analyses were performed using a pooled safety population, defined as all participants from the three DBPC trials who were randomized to treatment, received one or more doses of the study drug, and had one or more post-baseline safety assessment. Data were analyzed for the 6-week DBPC period with participants classified into three subgroups based on valbenazine dosage: pooled $40 \mathrm{mg} / \mathrm{day}$, which included doses of $40 \mathrm{mg}$ (KINECT 3) and $50 \mathrm{mg}$ (KINECT, KINECT 2); pooled $80 \mathrm{mg} /$ day, which included doses of $75 \mathrm{mg}$ (KINECT 2) and $80 \mathrm{mg}$ (KINECT 3); and placebo. Analyses of longer treatment with valbenazine (40 or $80 \mathrm{mg} / \mathrm{day}$ ) were conducted using data from the safety population of the valbenazine extension period of KINECT 3.

Outcomes of interest were treatment-emergent adverse events (TEAEs), vital sign measurements, and ECG parameters. The incidence of cardiac-related TEAEs was analyzed by aggregating the following cardiac-related MedDRA $^{\circledR}$ (Version 12.0) preferred TEAE terms: cardiac failure, chest pain, ECG QT prolonged, myocardial infarction, sudden death, and syncope and using the standardized MedDRA ${ }^{\circledR}$ query of torsades de pointes/QT prolongation. Additionally, any preferred term under the MedDRA ${ }^{\circledR}$ system organ class for "Cardiac Disorders" was included for the current analysis. The incidence of hypotension or orthostatic hypotension TEAEs was analyzed by aggregating the following MedDRA ${ }^{\circledR}$ preferred terms: blood pressure decreased, dizziness, dizziness postural, fall, hypotension, orthostatic hypotension, orthostatic intolerance, presyncope, and syncope.

Vital sign measurements included supine and orthostatic systolic blood pressure, diastolic blood pressure (DBP), and heart rate. The mean changes from baseline to week 6 were analyzed without any imputation for missing values. Additionally, orthostatic blood pressure measurements were analyzed for potentially clinically significant (PCS) values, defined as a decrease from baseline in systolic blood pressure of $\geq 20 \mathrm{mmHg}$ and a decrease in DBP of $\geq 10 \mathrm{mmHg}$.

Triplicate ECGs with machine-read parameters were used in the valbenazine clinical trials; analyses were based on the average of triplicate readings at each study visit. Mean changes from baseline to week 6 in ECG parameters were analyzed without any imputation for missing values. Two sets of categorical summaries for QTcF intervals were performed: (1) number and percentage of participants in each treatment group whose highest reported QTcF value met thresholds of $>450,>480$, or $>500 \mathrm{~ms}$; and (2) number and percentage of participants in each treatment group whose largest QTcF increase from baseline met thresholds of $>30$ or $>60 \mathrm{~ms}$.

The vital sign and ECG outcomes described above were also analyzed in the following subgroups from the pooled safety population: age $(<65, \geq 65$ years), sex (men, women), race (white, black or African American, other), body mass index $\left(<18.5,18.5\right.$ to $<25 \mathrm{~kg} / \mathrm{m}^{2}, 25$ to $<30$, $\geq 30 \mathrm{~kg} / \mathrm{m}^{2}$ ), known cardiac history (yes, no), psychiatric diagnosis (schizophrenia or schizoaffective disorder, mood disorder), antipsychotic medication use (atypical only, typical only, both, none), and use of any medication with a potential for QT prolongation (yes, no) [21].

Statistical analyses were conducted for the pooled DBPC studies. Mean changes from baseline (i.e., vital signs, ECG parameters) were analyzed using an analysis of covariance model that included groups, study, and baseline value as covariates. Categorical outcomes (i.e., TEAEs, PCS vital signs) were analyzed using the Fisher's exact test. For QTc categories, Fisher's exact test was conducted for any QTc value $\geq 450 \mathrm{~ms}$ and for any increase $\geq 30 \mathrm{~ms}$ from baseline. No statistical testing was conducted in the subgroups because of small sample sizes. For the KINECT 3 extension study, no statistical comparison was made between valbenazine dose groups because participants were allowed a one-time dose reduction for tolerability.

\section{Results}

\subsection{Demographics and Baseline Characteristics}

The pooled safety population included 400 participants (placebo, $n=178$; valbenazine $40 \mathrm{mg} /$ day, $n=110$; valbenazine $80 \mathrm{mg} / \mathrm{day}, n=112$ ). Demographics and baseline characteristics were generally similar across treatment groups (Table 1). The mean age in this population was 55.8 years, and the majority of participants were male $(57.3 \%)$, white $(55.5 \%)$, aged younger than 65 years $(82.8 \%)$, and had a diagnosis of schizophrenia or schizoaffective disorder (71.5\%). The mean age of TD diagnosis was 48.2 years.

Of the 400 participants in the pooled safety population, $388(97.0 \%)$ had some reported medical history (Table 1). A diagnosis of a specific cardiac disorder (per MedDRA ${ }^{\circledR}$ SOC) was found in $47(11.8 \%)$ participants. The most common terms reported as medical history $(>30 \%$ of all participants, based on MedDRA ${ }^{\circledR}$ preferred term) were hypertension $(53.3 \%)$, insomnia $(35.5 \%)$, and gastroesophageal reflux disease $(31.5 \%)$. In addition to their current psychiatric diagnosis, one-quarter of the population also reported a history of anxiety (25.5\%). Of all current 
Table 1 Demographics and baseline characteristics (pooled safety population)

\begin{tabular}{|c|c|c|c|}
\hline & $\begin{array}{l}\text { Placebo } \\
(n=178)\end{array}$ & $\begin{array}{l}\text { Valbenazine } 40 \mathrm{mg} / \text { day } \\
(n=110)\end{array}$ & $\begin{array}{l}\text { Valbenazine } 80 \mathrm{mg} / \text { day } \\
(n=112)\end{array}$ \\
\hline \multicolumn{4}{|l|}{ Age (years) } \\
\hline Mean \pm SD & $55.8 \pm 10.5$ & $55.4 \pm 8.5$ & $56.3 \pm 10.3$ \\
\hline$<65, n(\%)$ & $144(80.9)$ & $95(86.4)$ & $92(82.1)$ \\
\hline$\geq 65, n(\%)$ & $34(19.1)$ & 15 (13.6) & $20(17.9)$ \\
\hline \multicolumn{4}{|l|}{ Sex, $n(\%)$} \\
\hline Men & $102(57.3)$ & $66(60.0)$ & $61(54.5)$ \\
\hline Women & $76(42.7)$ & $44(40.0)$ & $51(45.5)$ \\
\hline \multicolumn{4}{|l|}{ Race, $n(\%)$} \\
\hline White & $95(53.4)$ & $62(56.4)$ & $65(58.0)$ \\
\hline Black/African American & $74(41.6)$ & $42(38.2)$ & $44(39.3)$ \\
\hline \multicolumn{4}{|l|}{ Body mass index $\left(\mathrm{kg} / \mathrm{m}^{2}\right)$} \\
\hline Mean \pm SD & $28.3 \pm 5.5$ & $28.8 \pm 5.6$ & $28.1 \pm 5.9$ \\
\hline$<18.5, n(\%)$ & $2(1.1)$ & $0(0)$ & $5(4.5)$ \\
\hline 18.5 to $<25, n(\%)$ & $47(26.4)$ & $29(26.4)$ & $32(28.6)$ \\
\hline 25 to $<30, n(\%)$ & $61(34.3)$ & $38(34.5)$ & $32(28.6)$ \\
\hline$\geq 30, n(\%)$ & $68(38.2)$ & $43(39.1)$ & $43(38.4)$ \\
\hline Age at $\mathrm{TD}$ diagnosis, mean $\pm \mathrm{SD}$ (years ${ }^{\mathrm{a}}$ ) & $48.4 \pm 11.9$ & $48.1 \pm 9.8$ & $48.0 \pm 12.3$ \\
\hline AIMS total score, mean $\pm \mathrm{SD}^{\mathrm{b}}$ & $9.0 \pm 4.5$ & $9.4 \pm 4.3$ & $9.6 \pm 3.6$ \\
\hline \multicolumn{4}{|l|}{ Vital signs, mean $\pm \mathrm{SD}$} \\
\hline Supine SBP (mmHg) & $122.6 \pm 13.3$ & $123.1 \pm 13.1$ & $123.9 \pm 13.4$ \\
\hline Supine DBP (mmHg) & $77.1 \pm 9.0$ & $78.3 \pm 8.0$ & $77.1 \pm 8.6$ \\
\hline Supine heart rate (bpm) & $76.5 \pm 11.4$ & $75.3 \pm 12.3$ & $74.4 \pm 12.0$ \\
\hline Orthostatic SBP $\left(\mathrm{mmHg}^{\mathrm{c}}\right)$ & $0.5 \pm 8.7$ & $-0.3 \pm 7.9$ & $-0.9 \pm 8.5$ \\
\hline Orthostatic DBP $\left(\mathrm{mmHg}^{\mathrm{c}}\right)$ & $2.0 \pm 7.3$ & $1.7 \pm 5.7$ & $0.9 \pm 6.1$ \\
\hline Orthostatic heart rate $\left(\mathrm{bpm}^{\mathrm{c}}\right)$ & $5.5 \pm 7.4$ & $5.0 \pm 6.4$ & $5.2 \pm 7.3$ \\
\hline \multicolumn{4}{|l|}{ Electrocardiogram, mean \pm SD } \\
\hline Heart rate $(\mathrm{bpm})$ & $75.2 \pm 13.2$ & $73.3 \pm 13.0$ & $74.3 \pm 13.6$ \\
\hline PR interval (ms) & $158.4 \pm 22.6$ & $157.1 \pm 25.9$ & $153.7 \pm 24.0$ \\
\hline QRS duration (ms) & $92.2 \pm 15.8$ & $90.0 \pm 10.6$ & $90.8 \pm 11.6$ \\
\hline QTcF interval (ms) & $412.0 \pm 20.3$ & $414.5 \pm 20.6$ & $412.9 \pm 21.9$ \\
\hline \multicolumn{4}{|l|}{ Medical history } \\
\hline Any medical history, $n(\%)$ & $172(96.6)$ & $107(97.3)$ & $109(97.3)$ \\
\hline Any cardiac disorder, $n(\%)^{\mathrm{d}}$ & $20(11.2)$ & $13(11.8)$ & $14(12.5)$ \\
\hline Coronary artery disease & $8(4.5)$ & $5(4.5)$ & $3(2.7)$ \\
\hline Myocardial infarction & $4(2.2)$ & $1(0.9)$ & $3(2.7)$ \\
\hline Cardiac failure congestive & $1(0.6)$ & $0(0.0)$ & $3(2.7)$ \\
\hline Arrhythmia & $2(1.1)$ & $0(0.0)$ & $2(1.8)$ \\
\hline Chest pain & $1(0.6)$ & $1(0.9)$ & $0(0.0)$ \\
\hline Angina pectoris & $3(1.7)$ & $1(0.9)$ & $0(0.0)$ \\
\hline Cardiac failure & $1(0.6)$ & $0(0.0)$ & $0(0.0)$ \\
\hline \multicolumn{4}{|l|}{ Other medical history, $n(\%)^{\mathrm{e}}$} \\
\hline Hypertension & $102(57.3)$ & $56(50.9)$ & $55(49.1)$ \\
\hline Insomnia & $61(34.3)$ & $38(34.5)$ & $43(38.4)$ \\
\hline Gastroesophageal reflux disease & $45(25.3)$ & $43(39.1)$ & $38(33.9)$ \\
\hline Anxiety & $45(25.3)$ & $30(27.3)$ & $27(24.1)$ \\
\hline Hypercholesterolemia & $34(19.1)$ & $18(16.4)$ & $31(27.7)$ \\
\hline Depression & $30(16.9)$ & $23(20.9)$ & $20(17.9)$ \\
\hline Chronic obstructive pulmonary disease & $25(14.0)$ & $19(17.3)$ & $20(17.9)$ \\
\hline
\end{tabular}


Table 1 continued

\begin{tabular}{|c|c|c|c|}
\hline & $\begin{array}{l}\text { Placebo } \\
(n=178)\end{array}$ & $\begin{array}{l}\text { Valbenazine } 40 \mathrm{mg} / \text { day } \\
(n=110)\end{array}$ & $\begin{array}{l}\text { Valbenazine } 80 \mathrm{mg} / \text { day } \\
(n=112)\end{array}$ \\
\hline Type 2 diabetes mellitus & $32(18.0)$ & $19(17.3)$ & $9(8.0)$ \\
\hline Drug hypersensitivity & $24(13.5)$ & $20(18.2)$ & $15(13.4)$ \\
\hline Hyperlipidemia & $28(15.7)$ & $15(13.6)$ & $15(13.4)$ \\
\hline Asthma & $23(12.9)$ & $9(8.2)$ & $20(17.9)$ \\
\hline Osteoarthritis & $21(11.8)$ & $16(14.5)$ & $15(13.4)$ \\
\hline Back pain & $22(12.4)$ & $16(14.5)$ & $14(12.5)$ \\
\hline Hypothyroidism & $22(12.4)$ & $12(10.9)$ & $14(12.5)$ \\
\hline Diabetes mellitus & $21(11.8)$ & $9(8.2)$ & $8(7.1)$ \\
\hline Seasonal allergy & $12(6.7)$ & $11(10.0)$ & $10(8.9)$ \\
\hline Drug abuse & $11(6.2)$ & $9(8.2)$ & $12(10.7)$ \\
\hline Hepatitis C & $10(5.6)$ & $11(10.0)$ & $8(7.1)$ \\
\hline Hysterectomy ${ }^{\mathrm{f}}$ & $19(25.0)$ & $9(20.5)$ & $8(15.7)$ \\
\hline \multicolumn{4}{|l|}{ CYP2D6 genotype, $n(\%)$} \\
\hline Poor metabolizer & $7(3.9)$ & $8(7.3)$ & $4(3.6)$ \\
\hline $\begin{array}{l}\text { Intermediate, extensive, or ultra-rapid } \\
\text { metabolizer }\end{array}$ & $171(96.1)$ & $101(91.8)$ & $107(95.5)$ \\
\hline Not reported & $0(0)$ & $1(0.9)$ & $1(0.9)$ \\
\hline \multicolumn{4}{|l|}{ Psychiatric history } \\
\hline \multicolumn{4}{|l|}{ Primary diagnosis, $n(\%)$} \\
\hline Schizophrenia or schizoaffective disorder & $134(75.3)$ & $82(74.5)$ & $70(62.5)$ \\
\hline Mood disorder & $44(24.7)$ & $28(25.5)$ & $42(37.5)$ \\
\hline Duration of illness, mean \pm SD (years) & $24.9 \pm 13.5$ & $23.3 \pm 13.0$ & $22.5 \pm 13.0$ \\
\hline
\end{tabular}

AIMS Abnormal Involuntary Movement Scale, $C Y P$ cytochrome P450, DBP diastolic blood pressure, $S B P$ systolic blood pressure, $T D$ tardive dyskinesia, $S O C$ system organ class, $S D$ standard deviation

${ }^{a}$ Based on available history: placebo, $n=138 ; 40 \mathrm{mg} /$ day, $n=85 ; 80 \mathrm{mg} / \mathrm{day}, n=82$

${ }^{\mathrm{b}}$ Based on available blinded central rater scores: placebo, $n=165 ; 40 \mathrm{mg} / \mathrm{day}, n=105 ; 80 \mathrm{mg} / \mathrm{day}, n=110$

${ }^{\mathrm{c} O r t h o s t a t i c ~ v i t a l ~ s i g n ~ m e a s u r e m e n t s ~ c a l c u l a t e d ~ a s ~ s t a n d i n g ~ m i n u s ~ s u p i n e ~ v a l u e s ~}$

${ }^{\mathrm{d}}$ Table lists all MedDRA ${ }^{\circledR}$ preferred terms for the SOC of cardiac disorder that were reported in any participant regardless of treatment

${ }^{\mathrm{e}}$ Table lists all MedDRA ${ }^{\circledR}$ preferred terms reported in $\geq 10 \%$ of participants in any treatment group

${ }^{\mathrm{f}}$ Based on the number of women in each treatment group: placebo, $n=76 ; 40 \mathrm{mg}, n=44 ; 80 \mathrm{mg}, n=51$

concomitant medications, the most common classes of drugs were antipsychotic (84.0\%), antidepressant (63.8\%), and anxiolytic (28.8\%) medications (Table 2). The majority of participants $(74.3 \%)$ were taking a concomitant medication with known potential to increase the QT interval, including some antipsychotic medications (e.g., risperidone, aripiprazole, olanzapine) and antidepressant medications (e.g., citalopram, mirtazapine).

\subsection{Treatment-Emergent Adverse Events}

Few participants in the valbenazine trials had cardiac-related TEAEs, and no statistically significant difference between valbenazine and placebo was found for any of these events (Table 3). Five cardiac-related TEAEs occurred during the 6-week DBPC period, each reported in one participant: chest pain $(40 \mathrm{mg} /$ day $)$; bradycardia (40 mg/day), blood pressure increased ( $80 \mathrm{mg} /$ day), myocardial infarction which resulted in death (placebo); and sudden death as a result of a possible cardiovascular event in a 73-year-old African American man with multiple cardiac risk factors ( $80 \mathrm{mg} /$ day) [17]. Both deaths were considered unrelated (placebo) or unlikely related $(80 \mathrm{mg} /$ day) to the study drug. During the valbenazine extension period of KINECT 3, cardiac-related TEAEs that occurred in more than one participant were chest pain $(40$ and $80 \mathrm{mg} /$ day, $n=2 \mathrm{each})$ and syncope $(40 \mathrm{mg} /$ day, $n=3 ; 80 \mathrm{mg} /$ day, $n=1)$. Cardiac-related TEAEs that led 
Table 2 Concomitant medications (pooled safety population)

\begin{tabular}{|c|c|c|c|}
\hline Concomitant medication, $n(\%)$ & $\begin{array}{l}\text { Placebo } \\
(n=178)\end{array}$ & $\begin{array}{l}\text { Valbenazine } \\
40 \mathrm{mg} / \text { day }(n=110)\end{array}$ & $\begin{array}{l}\text { Valbenazine } \\
80 \mathrm{mg} / \text { day }(n=112)\end{array}$ \\
\hline Any concomitant medication & $178(100.0)$ & $109(99.1)$ & $112(100.0)$ \\
\hline Any antipsychotic medication ${ }^{\mathrm{a}}$ & $149(83.7)$ & $98(89.1)$ & $89(79.5)$ \\
\hline Atypical only & $115(64.6)$ & $73(66.4)$ & $68(60.7)$ \\
\hline Typical or both & $34(19.1)$ & $24(21.8)$ & $18(16.1)$ \\
\hline Quetiapine $^{\mathrm{b}}$ & $39(21.9)$ & $31(28.2)$ & $23(20.5)$ \\
\hline Risperidone $^{\mathrm{b}}$ & $32(18.0)$ & $13(11.8)$ & $19(17.0)$ \\
\hline Haloperidol $^{\mathrm{b}}$ & $15(8.4)$ & $16(14.5)$ & 13 (11.6) \\
\hline Aripiprazole ${ }^{\mathrm{b}}$ & $20(11.2)$ & $12(10.9)$ & 15 (13.4) \\
\hline Olanzapine $^{\mathrm{b}}$ & $21(11.8)$ & $15(13.6)$ & $10(8.9)$ \\
\hline Ziprasidone $^{\mathrm{b}}$ & $6(3.4)$ & $6(5.5)$ & $11(9.8)$ \\
\hline Lithium $^{\mathrm{b}}$ & $2(1.1)$ & $6(5.5)$ & $10(8.9)$ \\
\hline Paliperidone $^{\mathrm{b}}$ & $3(1.7)$ & $7(6.4)$ & $3(2.7)$ \\
\hline Lurasidone & $5(2.8)$ & $6(5.5)$ & $3(2.7)$ \\
\hline Perphenazine $^{\mathrm{b}}$ & $6(3.4)$ & $7(6.4)$ & $2(1.8)$ \\
\hline Clozapine $^{\mathrm{b}}$ & $10(5.6)$ & $3(2.7)$ & $3(2.7)$ \\
\hline Any antidepressant medication ${ }^{\mathrm{a}}$ & $111(62.4)$ & $73(66.4)$ & $71(63.4)$ \\
\hline Trazodone $^{\mathrm{b}}$ & $32(18.0)$ & $26(23.6)$ & $23(20.5)$ \\
\hline Citalopram $^{\mathrm{b}}$ & $23(12.9)$ & $16(14.5)$ & 13 (11.6) \\
\hline Sertraline $^{\mathrm{b}}$ & $23(12.9)$ & $13(11.8)$ & $14(12.5)$ \\
\hline Mirtazapine $^{\mathrm{b}}$ & $12(6.7)$ & $10(9.1)$ & $7(6.3)$ \\
\hline Buproprion & $10(5.6)$ & $8(7.3)$ & $8(7.1)$ \\
\hline Escitalopram $^{\mathrm{b}}$ & $5(2.8)$ & $7(6.4)$ & $7(6.3)$ \\
\hline Duloxetine & $6(3.4)$ & $6(5.5)$ & $6(5.4)$ \\
\hline Fluoxetine $^{\mathrm{b}}$ & $12(6.7)$ & $7(6.4)$ & $4(3.6)$ \\
\hline Any anxiolytic medication ${ }^{a}$ & $49(27.5)$ & $40(36.4)$ & $26(23.2)$ \\
\hline Lorazepam & $18(10.1)$ & $12(10.9)$ & $13(11.6)$ \\
\hline Hydroxyzine $^{\mathrm{b}}$ & $14(7.9)$ & $10(9.1)$ & $9(8.0)$ \\
\hline Alprazolam & 7 (3.9) & $13(11.8)$ & $4(3.6)$ \\
\hline Buspirone & $6(3.4)$ & $8(7.3)$ & $4(3.6)$ \\
\hline \multicolumn{4}{|l|}{ Cardiovascular medications $^{\mathrm{c}}$} \\
\hline Lisinopril & $46(25.8)$ & $16(14.5)$ & $21(18.8)$ \\
\hline Amlodipine & $24(13.5)$ & $11(10.0)$ & $12(10.7)$ \\
\hline Hydrochlorothiazide ${ }^{\mathrm{b}}$ & $10(5.6)$ & $9(8.2)$ & $9(8.0)$ \\
\hline Metoprolol & $10(5.6)$ & $7(6.4)$ & $3(2.7)$ \\
\hline Losartan & $8(4.5)$ & $3(2.7)$ & $4(3.6)$ \\
\hline Furosemide $^{b}$ & $5(2.8)$ & $3(2.7)$ & $4(3.6)$ \\
\hline Propranolol & $4(2.2)$ & $5(4.5)$ & $1(0.9)$ \\
\hline Enalapril & $3(1.7)$ & $4(3.6)$ & $1(0.9)$ \\
\hline Carvedilol & $3(1.7)$ & $3(2.7)$ & $0(0.0)$ \\
\hline Atenolol & $4(2.2)$ & $0(0.0)$ & $2(1.8)$ \\
\hline Clonidine & $3(1.7)$ & $0(0.0)$ & $3(2.7)$ \\
\hline Nifedipine & $2(1.1)$ & $3(2.7)$ & $0(0.0)$ \\
\hline \multicolumn{4}{|l|}{ Diabetes mellitus medications ${ }^{\mathrm{d}}$} \\
\hline Metformin & $43(24.2)$ & $20(18.2)$ & $15(13.4)$ \\
\hline Insulin glargine & $8(4.5)$ & $4(3.6)$ & $5(4.5)$ \\
\hline Glipizide & $4(2.2)$ & $2(1.8)$ & $2(1.8)$ \\
\hline Sitagliptin & $3(1.7)$ & $4(3.6)$ & $1(0.9)$ \\
\hline
\end{tabular}


Table 2 continued

\begin{tabular}{lccc}
\hline Concomitant medication, $n(\%)$ & $\begin{array}{l}\text { Placebo } \\
(n=178)\end{array}$ & $\begin{array}{c}\text { Valbenazine } \\
40 \mathrm{mg} / \text { day }(n=110)\end{array}$ & $\begin{array}{c}\text { Valbenazine } \\
80 \mathrm{mg} / \mathrm{day}(n=112)\end{array}$ \\
\hline Glibenclamide & $1(0.6)$ & $4(3.6)$ & $1(0.9)$ \\
Liraglutide & $1(0.6)$ & $3(2.7)$ & $10.9)$ \\
Lipid-modifying agents ${ }^{\mathrm{e}}$ & & & $10(8.9)$ \\
Simvastatin & $22(12.4)$ & $9(8.2)$ & $11(9.8)$ \\
Atorvastatin & $10(5.6)$ & $9(8.2)$ & $1(0.9)$ \\
Pravastatin & $9(5.1)$ & $8(7.3)$ & $5(4.5)$ \\
Rosuvastatin & $8(4.5)$ & $4(3.6)$ & $4(3.6)$ \\
Fenofibrate & $6(3.4)$ & $4(3.6)$ & $3(2.7)$ \\
Omega-3 fatty acids & $4(2.2)$ & $2(1.8)$ & $2(1.8)$ \\
Gemfibrozil & $4(2.2)$ & $1(0.9)$ & $2(1.8)$ \\
Lovastatin & $4(2.2)$ & $1(0.9)$ & $80(71.4)$ \\
Any medication with the potential & $130(73.0)$ & $87(79.1)$ & \\
to prolong the QT interval ${ }^{\mathrm{b}}$ & & & \\
\hline
\end{tabular}

${ }^{a}$ Based on specific World Health Organization Drug Anatomical Therapeutic Chemical Category (i.e., antipsychotics, antidepressants, anxiolytics); individual medications include all drugs in these categories that were used in $\geq 5 \%$ of participants in any treatment group

${ }^{\mathrm{b}}$ Medications with potential to prolong the QT interval [21]

${ }^{\mathrm{c}}$ Based on multiple drug categories; individual medications include all acetylcholinesterase inhibitors, angiotensin antagonists, antiadrenergic agents, antiarrhythmic agents, beta blockers, cardiac glycosides, diuretics, potassium-sparing agents, selective calcium channel blockers, and vasodilators that were used in $\geq 2 \%$ of participants in any treatment group

${ }^{\mathrm{d}}$ Based on multiple drug categories; individual medications include all blood glucose-lowering agents, glycogenolytic hormones, insulins, and insulin analogs that were used in $\geq 2 \%$ of participants in any treatment group

${ }^{\mathrm{e}}$ Based on multiple drug categories; individual medications include all lipid-modifying agents (simple or combinations) that were used in $\geq 2 \%$ of participants in any treatment group

to discontinuation from the KINECT 3 valbenazine extension period were syncope (40 and $80 \mathrm{mg} / \mathrm{day}, n=1$ each) and cardiac failure ( $80 \mathrm{mg} /$ day, $n=1)$.

The TEAEs evaluated as potentially related to hypotension also included orthostatic hypotension, dizziness, and fall (Table 3). None of these TEAEs resulted in discontinuation from the DBPC trials or the KINECT 3 valbenazine extension period.

\subsection{Vital Signs}

Mean values at baseline for supine and orthostatic vital sign measurements are shown in Table 1. Mean changes from baseline to week 6 (pooled safety population) and week 48 (KINECT 3 extension safety population) in vital sign measurements were generally small and similar across treatment groups (Table 4). No statistically significant difference between valbenazine and placebo was found in any week 6 outcome, except for a mean increase in orthostatic DBP (40 mg/day, $1.3 \pm 8.4 \mathrm{mmHg}$ ).

During the 6-week DBPC trials, $<5 \%$ of participants in all treatment groups met the PCS criteria for low orthostatic systolic blood pressure (decrease $\geq 20 \mathrm{mmHg}$ : placebo, 3.8\%; $40 \mathrm{mg} /$ day, 3.1\%; $80 \mathrm{mg} / \mathrm{day}, 2.0 \%$ ) and DBP (decrease $\geq 10 \mathrm{mmHg}$ : placebo, $3.8 \%$; $40 \mathrm{mg} /$ day, $3.1 \%$; $80 \mathrm{mg} / \mathrm{day}, 3.0 \%$ ). The rates for increases in orthostatic heart rates were similar among treatment groups (increase $\geq 15 \mathrm{bpm}$ : placebo, $10.7 \%$; $40 \mathrm{mg} / \mathrm{day}, 9.4 \%$; $80 \mathrm{mg} /$ day, $15.8 \%$ ). No statistical difference between valbenazine and placebo was found in any of these outcomes. Subgroup analyses indicated no apparent effects of demographics or other baseline characteristics on vital signs (data not shown).

\subsection{Electrocardiogram Parameters}

Mean values at baseline for ECG parameters are shown in Table 1. Mean changes from baseline to week 6 (pooled safety population) and week 48 (KINECT 3 extension safety population) were generally small and not considered clinically important (Table 5), with no statistically significant difference between valbenazine and placebo except for a mean decrease in ECG heart rate $(80 \mathrm{mg} /$ day, $-3.4 \pm 10.6 \mathrm{bpm})$. At week 6 , mean changes in QTcF were similar across treatment groups: placebo, $1.3 \mathrm{~ms}$; $40 \mathrm{mg} /$ day, $1.1 \mathrm{~ms} ; 80 \mathrm{mg} /$ day, $2.1 \mathrm{~ms}$; all $p>0.05$. 
Table 3 Treatment-emergent adverse events

\begin{tabular}{|c|c|c|c|c|c|}
\hline \multirow[t]{2}{*}{$\begin{array}{l}\text { MedDRA }{ }^{\circledR} \text { preferred } \\
\text { term, } n(\%)\end{array}$} & \multicolumn{3}{|c|}{ Double-blind placebo-controlled trials (pooled safety population) } & \multicolumn{2}{|c|}{$\begin{array}{l}\text { KINECT } 3 \text { valbenazine extension period (safety } \\
\text { population) }\end{array}$} \\
\hline & $\begin{array}{l}\text { Placebo } \\
(n=178)\end{array}$ & $\begin{array}{l}\text { Valbenazine } \\
40 \mathrm{mg} / \text { day }^{\mathrm{a}}(n=110)\end{array}$ & $\begin{array}{l}\text { Valbenazine } \\
80 \mathrm{mg} / \text { day }^{\mathrm{a}}(n=112)\end{array}$ & $\begin{array}{l}\text { Valbenazine } \\
40 \mathrm{mg} / \text { day }(n=97)\end{array}$ & $\begin{array}{l}\text { Valbenazine } 80 \mathrm{mg} / \text { day } \\
(n=101)\end{array}$ \\
\hline \multicolumn{6}{|l|}{ Cardiac-related TEAEs ${ }^{\mathrm{b}}$} \\
\hline Chest pain & $0(0)$ & $1(0.9)$ & $0(0)$ & $2(2.1)$ & $2(2.0)$ \\
\hline Bradycardia & $0(0)$ & $1(0.9)$ & $0(0)$ & $0(0)$ & $0(0)$ \\
\hline $\begin{array}{l}\text { Blood pressure } \\
\text { increased }\end{array}$ & $0(0)$ & $0(0)$ & $1(0.9)$ & $0(0)$ & $1(1.0)$ \\
\hline Sudden death & $0(0)$ & $0(0)$ & $1(0.9)$ & $0(0)$ & $0(0)$ \\
\hline $\begin{array}{l}\text { Myocardial } \\
\text { infarction }\end{array}$ & $1(0.6)$ & $0(0)$ & $0(0)$ & $0(0)$ & $0(0)$ \\
\hline Syncope ${ }^{c}$ & $0(0)$ & $0(0)$ & $0(0)$ & $3(3.1)$ & $1(1.0)$ \\
\hline Cardiac failure & $0(0)$ & $0(0)$ & $0(0)$ & $0(0)$ & $1(1.0)$ \\
\hline $\begin{array}{l}\text { Bundle branch block } \\
\text { left }\end{array}$ & $0(0)$ & $0(0)$ & $0(0)$ & $0(0)$ & $1(1.0)$ \\
\hline $\begin{array}{l}\text { Mitral valve } \\
\text { incompetence }\end{array}$ & $0(0)$ & $0(0)$ & $0(0)$ & $0(0)$ & $1(1.0)$ \\
\hline $\begin{array}{l}\text { Tricuspid valve } \\
\text { incompetence }\end{array}$ & $0(0)$ & $0(0)$ & $0(0)$ & $0(0)$ & $1(1.0)$ \\
\hline $\begin{array}{l}\text { Arrhythmia } \\
\text { supraventricular }\end{array}$ & $0(0)$ & $0(0)$ & $0(0)$ & $1(1.0)$ & $0(0)$ \\
\hline $\begin{array}{l}\text { Coronary artery } \\
\text { disease }\end{array}$ & $0(0)$ & $0(0)$ & $0(0)$ & $1(1.0)$ & $0(0)$ \\
\hline Sinus tachycardia & $0(0)$ & $0(0)$ & $0(0)$ & $1(1.0)$ & $0(0)$ \\
\hline $\begin{array}{l}\text { Supraventricular } \\
\text { tachycardia }\end{array}$ & $0(0)$ & $0(0)$ & $0(0)$ & $1(1.0)$ & $0(0)$ \\
\hline \multicolumn{6}{|c|}{ Hypotension-related TEAEs ${ }^{\mathrm{d}}$} \\
\hline Dizziness & $4(2.2)$ & $2(1.8)$ & $0(0)$ & $4(4.1)$ & $7(6.9)$ \\
\hline Fall & $0(0)$ & $2(1.8)$ & $1(0.9)$ & $3(3.1)$ & $2(2.0)$ \\
\hline Hypotension & $0(0)$ & $0(0)$ & $1(0.9)$ & $0(0)$ & $1(1.0)$ \\
\hline $\begin{array}{l}\text { Orthostatic } \\
\text { hypotension }\end{array}$ & $0(0)$ & $0(0)$ & $0(0)$ & $1(1.0)$ & $0(0)$ \\
\hline
\end{tabular}

SOC system organ class, TEAEs treatment-emergent adverse events

${ }^{a}$ No significant difference found between valbenazine $(40$ or $80 \mathrm{mg}$ ) and placebo for any TEAE presented in this table

${ }^{\mathrm{b}}$ Includes any of the aggregated MedDRA ${ }^{\circledR}$ preferred terms (cardiac failure, chest pain, electrocardiogram QT prolonged, myocardial infarction, sudden death, and syncope), standardized MedDRA ${ }^{\circledR}$ query of torsades de pointes/QT prolongation, and preferred term under the MedDRA ${ }^{\circledR}$ SOC for Cardiac Disorders

${ }^{\mathrm{c}}$ Syncope included in the aggregated terms for both cardiac-related and hypotension-related TEAEs

${ }^{\mathrm{d}}$ Includes any of the aggregated MedDRA ${ }^{\circledR}$ preferred terms for hypotension or orthostatic hypotension (blood pressure decreased, dizziness, dizziness postural, fall, hypotension, orthostatic hypotension, orthostatic intolerance, presyncope, and syncope)

Categorical analyses showed that although QTcF values exceeded $450 \mathrm{~ms}$ in some participants during the 6-week DBPC period (Table 5), there was no apparent difference between placebo and valbenazine (40 or $80 \mathrm{mg} /$ day). During the KINECT 3 valbenazine extension period, more participants in the $80-\mathrm{mg} / \mathrm{day}$ group had a QTcF $>450 \mathrm{~ms}$ or a QTcF $>30 \mathrm{~ms}$ increase from baseline, as compared with the 40-mg/day group (Table 5). However, only a few participants had a QTcF $>480 \mathrm{~ms} \quad(n=5)$, QTcF
$>500 \mathrm{~ms}(n=1)$, or a QTcF increase $>60 \mathrm{~ms}(n=6)$ at any point during this extension study, with no apparent difference between dose groups.

Results from subgroup analyses indicated no apparent effects of any baseline characteristic on ECG parameters (data not shown). In the subgroup taking a concomitant medication with known potential to prolong the QT interval, mean QTcF values at baseline and week 6 were as follows (for placebo, valbenazine $40 \mathrm{mg} /$ day, and 
Table 4 Mean changes from baseline in supine and orthostatic vital sign measurements

\begin{tabular}{|c|c|c|c|c|c|c|c|c|c|c|}
\hline \multirow[t]{3}{*}{ Vital sign parameter } & \multicolumn{6}{|c|}{$\begin{array}{l}\text { Double-blind placebo-controlled trials at week } 6 \text { (pooled safety } \\
\text { population) }\end{array}$} & \multicolumn{4}{|c|}{$\begin{array}{l}\text { KINECT } 3 \text { valbenazine extension period at } \\
\text { week } 48 \text { (safety population) }\end{array}$} \\
\hline & \multicolumn{2}{|c|}{ Placebo } & \multicolumn{2}{|c|}{$\begin{array}{l}\text { Valbenazine } \\
40 \mathrm{mg} / \text { day }\end{array}$} & \multicolumn{2}{|c|}{$\begin{array}{l}\text { Valbenazine } \\
80 \mathrm{mg} / \text { day }\end{array}$} & \multicolumn{2}{|c|}{$\begin{array}{l}\text { Valbenazine } \\
40 \mathrm{mg} / \text { day }\end{array}$} & \multicolumn{2}{|c|}{$\begin{array}{l}\text { Valbenazine } \\
80 \mathrm{mg} / \text { day }\end{array}$} \\
\hline & $n$ & $\begin{array}{l}\text { Mean } \\
\mathrm{CFB} \pm \mathrm{SD}\end{array}$ & $n$ & $\begin{array}{l}\text { Mean } \\
\mathrm{CFB} \pm \mathrm{SD}\end{array}$ & $n$ & $\begin{array}{l}\text { Mean } \\
\mathrm{CFB} \pm \mathrm{SD}\end{array}$ & $n$ & $\begin{array}{l}\text { Mean } \\
\mathrm{CFB} \pm \mathrm{SD}\end{array}$ & $n$ & $\begin{array}{l}\text { Mean } \\
\mathrm{CFB} \pm \mathrm{SD}\end{array}$ \\
\hline Supine SBP (mmHg) & 159 & $0.2 \pm 12.8$ & 96 & $-2.1 \pm 12.7$ & 101 & $-1.8 \pm 15.3$ & 61 & $0.1 \pm 14.8$ & 63 & $-0.1 \pm 15.5$ \\
\hline Supine DBP (mmHg) & 159 & $-0.1 \pm 10.0$ & 96 & $-1.6 \pm 8.5$ & 101 & $-1.2 \pm 9.7$ & 61 & $0.6 \pm 10.0$ & 63 & $-1.3 \pm 10.2$ \\
\hline Supine heart rate (bpm) & 159 & $-1.7 \pm 8.9$ & 96 & $-2.2 \pm 12.1$ & 101 & $-1.7 \pm 11.4$ & 61 & $-1.9 \pm 11.8$ & 63 & $-0.4 \pm 11.3$ \\
\hline Orthostatic SBP (mmHg) & 159 & $-0.8 \pm 10.2$ & 96 & $0.6 \pm 10.9$ & 101 & $0.1 \pm 9.2$ & 61 & $1.1 \pm 11.3$ & 63 & $-0.2 \pm 8.7$ \\
\hline Orthostatic DBP (mmHg) & 159 & $-0.5 \pm 8.3$ & 96 & $1.3 \pm 8.4^{\mathrm{b}}$ & 101 & $1.1 \pm 8.4$ & 61 & $0.0 \pm 7.7$ & 63 & $1.2 \pm 10.1$ \\
\hline $\begin{array}{l}\text { Orthostatic heart rate } \\
\text { (bpm) }\end{array}$ & 159 & $-0.8 \pm 8.3$ & 96 & $0.0 \pm 7.9$ & 101 & $1.1 \pm 7.1$ & 61 & $0.6 \pm 8.8$ & 63 & $1.5 \pm 7.1$ \\
\hline
\end{tabular}

$C F B$ change from baseline, $D B P$ diastolic blood pressure, $n$ number of participants with available vital assessment at week 6 (pooled safety population) or week 48 (KINECT 3 extension safety population), SBP systolic blood pressure, SD standard deviation

${ }^{a}$ Orthostatic vital sign measurements calculated as standing minus supine values

${ }^{\mathrm{b}} p<0.05$ vs. placebo

Table 5 Electrocardiogram parameters

\begin{tabular}{|c|c|c|c|c|c|c|c|c|c|c|}
\hline \multirow[t]{3}{*}{$\begin{array}{l}\text { Electrocardiogram } \\
\text { parameter }\end{array}$} & \multicolumn{6}{|c|}{$\begin{array}{l}\text { Double-blind placebo-controlled trials at week } 6 \text { (pooled safety } \\
\text { population) }\end{array}$} & \multicolumn{4}{|c|}{$\begin{array}{l}\text { KINECT } 3 \text { valbenazine extension period at } \\
\text { week } 48 \text { (safety population) }\end{array}$} \\
\hline & \multicolumn{2}{|c|}{ Placebo } & \multicolumn{2}{|c|}{$\begin{array}{l}\text { Valbenazine } \\
40 \mathrm{mg} / \text { day }\end{array}$} & \multicolumn{2}{|c|}{$\begin{array}{l}\text { Valbenazine } \\
80 \mathrm{mg} / \text { day }\end{array}$} & \multicolumn{2}{|c|}{$\begin{array}{l}\text { Valbenazine } \\
40 \mathrm{mg} / \text { day }\end{array}$} & \multicolumn{2}{|c|}{$\begin{array}{l}\text { Valbenazine } \\
80 \mathrm{mg} / \text { day }\end{array}$} \\
\hline & $n$ & $\begin{array}{l}\text { Mean } \\
\mathrm{CFB} \pm \mathrm{SD}\end{array}$ & $n$ & $\begin{array}{l}\text { Mean } \\
\mathrm{CFB} \pm \mathrm{SD}\end{array}$ & $n$ & $\begin{array}{l}\text { Mean } \\
\mathrm{CFB} \pm \mathrm{SD}\end{array}$ & $n$ & $\begin{array}{l}\text { Mean } \\
\mathrm{CFB} \pm \mathrm{SD}\end{array}$ & $n$ & $\begin{array}{l}\text { Mean } \\
\mathrm{CFB} \pm \mathrm{SD}\end{array}$ \\
\hline Heart rate (bpm) & 158 & $-0.4 \pm 10.2$ & 96 & $-1.8 \pm 11.9$ & 101 & $-3.4 \pm 10.6^{\mathrm{a}}$ & 61 & $-2.4 \pm 11.8$ & 62 & $0.2 \pm 12.6$ \\
\hline PR interval (ms) & 156 & $-1.0 \pm 14.2$ & 95 & $1.6 \pm 10.7$ & 101 & $2.2 \pm 14.3$ & 59 & $-1.1 \pm 12.1$ & 61 & $5.5 \pm 14.7$ \\
\hline QRS duration (ms) & 158 & $0.1 \pm 6.8$ & 96 & $-1.0 \pm 6.0$ & 101 & $-0.8 \pm 7.7$ & 61 & $-0.3 \pm 6.4$ & 62 & $1.7 \pm 12.6$ \\
\hline QTcF interval (ms) & 158 & $1.3 \pm 13.8$ & 96 & $1.1 \pm 16.6$ & 101 & $2.1 \pm 15.0$ & 61 & $5.0 \pm 20.0$ & 62 & $3.7 \pm 18.2$ \\
\hline QTcF interval $^{\mathrm{b}}$ & & $n_{1}(\%)$ & & $n_{1}(\%)$ & & $n_{1}(\%)$ & & $n_{1}(\%)$ & & $n_{1}(\%)$ \\
\hline$>450 \mathrm{~ms}$ & 178 & $11(6.2)$ & 110 & $11(10.0)$ & 112 & $5(4.5)$ & 95 & $13(13.7)$ & 100 & $22(22.0)$ \\
\hline$>480 \mathrm{~ms}$ & 178 & $2(1.1)$ & 110 & $0(0)$ & 112 & $0(0)$ & 95 & $2(2.1)$ & 100 & $3(3.0)$ \\
\hline$>500 \mathrm{~ms}$ & 178 & $0(0)$ & 110 & $0(0)$ & 112 & $0(0)$ & 95 & $0(0)$ & 100 & $1(1.0)$ \\
\hline$>30 \mathrm{~ms}$ increase & 178 & $11(6.2)$ & 110 & $3(2.7)$ & 112 & $6(5.4)$ & 95 & $14(14.7)$ & 100 & $24(24.0)$ \\
\hline$>60 \mathrm{~ms}$ increase & 178 & $1(0.6)$ & 110 & $1(0.9)$ & 112 & $0(0)$ & 95 & $3(3.2)$ & 100 & $3(3.0)$ \\
\hline
\end{tabular}

$C F B$ change from baseline, $n$ number of participants with vital available assessment at week 6 (pooled safety population), week 48 (KINECT 3 extension safety population), or at any study visit (for all QTcF interval analyses), $n_{l}$ number of participants who met the QTcF interval threshold, $S D$ standard deviation

${ }^{a} p<0.05$ vs. placebo

${ }^{\mathrm{b}}$ Based on the highest value at any visit during double-blind treatment; participants only counted once in each threshold category. No significant difference between valbenazine ( $40 \mathrm{or} 80 \mathrm{mg}$ ) and placebo for any QTcF interval $\geq 450 \mathrm{~ms}$ or any increase $\geq 30 \mathrm{~ms}$

valbenazine $80 \mathrm{mg} /$ day, respectively): baseline (411.2, 414.0, and $413.0 \mathrm{~ms})$; week 6 (412.2, 413.7, and $413.6 \mathrm{~ms}$ ). Similar results were found in the subgroup not taking a concomitant QT-prolonging medication: baseline (414.1, 416.3, and 412.6 ms); week 6 (417.9, 418.7, and $418.3 \mathrm{~ms})$. 


\section{Discussion}

Analyses of data from 6-week DBPC trials showed no notable differences between valbenazine ( 40 or $80 \mathrm{mg} /$ day) and placebo in the incidence of cardiac- or hypotensionrelated TEAEs, mean changes in vital sign measurements, clinically relevant changes in orthostatic blood pressure or heart rate (per PCS criteria), or mean changes in ECG parameters. None of the DBPC outcomes were statistically significant, except for a mean increase in orthostatic DBP $(40 \mathrm{mg} /$ day) and a mean decrease in ECG heart rate ( $80 \mathrm{mg} /$ day). However, these changes were not considered clinically relevant because the magnitudes were notably smaller than the corresponding standard deviations and the directions of change were opposite of what would be expected with orthostatic hypotension. Mean changes in vital sign measurements and ECG parameters during longer treatment (i.e., KINECT 3 valbenazine extension period) were comparable to results from the DBPC trials.

Participants in the phase II/III clinical trials had a psychiatric diagnosis of schizophrenia or schizoaffective disorder, or a mood disorder, and concomitant treatment with antipsychotic and/or antidepressant medications (at stable doses) was allowed for the management of psychiatric disorders. In addition to their current psychiatric diagnosis, $11.8 \%$ of participants in the pooled safety population reported a prior cardiovascular diagnosis or event (e.g., coronary artery disease, myocardial infarction). The medical history of study participants also included the following potential risk factors for cardiovascular disease: hypertension (53.3\%), hypercholesterolemia (20.8\%), hyperlipidemia (14.5\%), type 2 diabetes mellitus (15.0\%), hypothyroidism $(12.0 \%)$, and diabetes mellitus (9.5\%). While the cardiovascular risk profile of this study population may differ somewhat from a more general population of patients with TD, owing to the exclusion of participants with an unstable medical condition or significant cardiac abnormality, the characteristics of the study participants enrolled included co-morbidities and complicated medication requirements that generally reflect a real-world patient population.

Because orthostatic hypotension has been associated with some antipsychotic and antidepressant medications [22], this was an outcome of interest in the current analyses. There were no discernable differences between valbenazine and placebo with regard to hypotension-related TEAEs, mean changes in orthostatic vital sign measurements, or PCS decreases in orthostatic blood pressure or heart rate. The incidences of some TEAEs (e.g., dizziness, fall) were slightly higher in the KINECT 3 valbenazine extension period than in the 6-week DBPC trials (Table 3), which may be expected with a longer duration of observation on treatment. The prescribing information for valbenazine does not include any precautionary statements regarding orthostatic hypotension [19].

The prescribing information for valbenazine only carries two warnings, one of which is the potential for QT prolongation. This warning was based on the Food and Drug Administration's modeling of data from phase I studies. The prescribing information clarifies that the degree of QT prolongation is not clinically significant at plasma concentrations expected with recommended dosing. However, dose reduction may be necessary in certain situations in which valbenazine exposure may be higher; additionally, avoiding use in patients with congenital long QT syndrome or arrhythmias associated with a prolonged QT interval is recommended [19]. Categorical analyses of the QTcF interval for the 6-week DBPC treatment period showed no clear difference between valbenazine and placebo (Table 5). However, as might be expected in a study of longer duration (i.e., KINECT 3 extension, which included participants who received up to 48 weeks of valbenazine treatment), more participants met QTcF interval categorical criteria at least once during treatment (i.e., QTcF $>450 \mathrm{~ms}$, QTcF increase $>30 \mathrm{~ms}$ ) in this study, but interpretation is limited by the lack of a placebo control group.

In addition, although the valbenazine trials excluded patients with a current QTcF of $>450 \mathrm{~ms}$ (men) or $>470 \mathrm{~ms}$ (women) or a history of QT prolongation, medications with a known potential for QT prolongation were allowed during the study based on a case-by-case medical review. In the pooled safety population, $74.3 \%$ of participants were taking a medication with QT prolongation potential, which included some of the antipsychotic and antidepressant medications that were required for psychiatric treatment. More importantly, results from our subgroup analyses suggest that the addition of valbenazine to any of these medications did not appear to exacerbate the risk for a prolonged QT interval.

Based on results from drug-drug interaction studies (manuscripts in preparation), dose adjustments are recommended in known cytochrome P450 (CYP)2D6 poor metabolizers, patients taking a strong CYP2D6 inhibitor (e.g., paroxetine, fluoxetine, quinidine), and patients taking a strong CYP3A4 inhibitor (e.g., itraconazole, ketoconazole, clarithromycin); concomitant treatment with strong CYP3A4 inducers is not recommended [19]. In the pooled phase II/III clinical trials, only $4.8 \%$ (19/400) of participants were known poor CYP2D6 metabolizers. Because it is difficult to interpret results in such a small sample, subgroup analyses based on CYP2D6 metabolism were not included in this report. Although genotyping for CYP2D6 metabolism is recommended for patients requiring higher doses of tetrabenazine (an older vesicular monoamine 
transporter 2 inhibitor) [23], CYP2D6 genotyping is not required for valbenazine [19].

The main limitations of this report are that the analyses were conducted post hoc and that the clinical trials were not specifically designed or powered to conduct a statistical assessment of cardiovascular parameters with valbenazine treatment; nor were the trials designed to be analyzed based on the subgroups defined in this report. In addition, adverse events in clinical trials may be affected by factors such as the baseline health of participants, changes in disease state over the course of treatment, and tolerance to treatment. The current analyses were not adjusted for these factors, although efforts were made to explore the potential effects of various baseline characteristics on cardiovascular outcomes (i.e., subgroup analyses). As mentioned earlier, exclusion criteria for the clinical trials may limit the generalizability of the results. However, the data represent a large sample of adults with TD who received valbenazine in addition to their psychiatric medications, and results of the analyses indicate that valbenazine treatment confers minimal cardiovascular risk.

\section{Conclusions}

The results of analyses presented in this report indicate that in DBPC clinical trials, the short-term cardiovascular effects of valbenazine were generally similar to those of placebo. No unexpected cardiovascular effects were found in a longer extension period in which participants received up to 48 weeks of treatment with valbenazine. Importantly, more than $70 \%$ of participants in the valbenazine studies were taking concomitant medications that have been associated with increased cardiovascular risks and/or QT prolongation, including antipsychotic and antidepressant medications needed to treat primary psychiatric disorders. Post-marketing surveillance, along with pending safety data from other longer studies [NCT02405091 (12 months), NCT02736955 (18 months)], will help to further clarify the cardiovascular effects of valbenazine in patients with TD. In addition, the cardiovascular effects of valbenazine will be evaluated in planned and ongoing studies of other movement disorders (e.g., Tourette syndrome).

Acknowledgements Writing and editorial assistance was provided by Prescott Medical Communications Group, Inc. (Chicago, IL, USA) with support from Neurocrine Biosciences, Inc. (San Diego, CA, USA).

\section{Compliance with Ethical Standards}

Funding This study was sponsored by Neurocrine Biosciences, Inc. (San Diego, CA, USA).
Conflicts of interest Dao Thai-Cuarto, Christopher F. O'Brien, Roland Jimenez, Grace S. Liang, and Joshua Burke are full-time employees of Neurocrine Biosciences, Inc. and have stock/stock options in the company.

Ethical approval All procedures performed in studies involving human participants were in accordance with the ethical standards of the institutional and/or national research committee and with the 1964 Helsinki Declaration and its later amendments or comparable ethical standards. The trials were also conducted in accordance with International Conference on Harmonization Guidelines for Good Clinical Practice, US Code of Federal Regulations standards, US Food and Drug Administration, and the Health Canada Guidance for Clinical Trial Sponsors. Study protocols were approved by institutional review boards at participating centers. Only subjects assessed as having the capacity to provide consent (using the University of California, San Diego Brief Assessment of Capacity to Consent) were allowed to participate in the clinical trials.

Open Access This article is distributed under the terms of the Creative Commons Attribution-NonCommercial 4.0 International License (http://creativecommons.org/licenses/by-nc/4.0/), which permits any noncommercial use, distribution, and reproduction in any medium, provided you give appropriate credit to the original author(s) and the source, provide a link to the Creative Commons license, and indicate if changes were made.

\section{References}

1. Carter P, Mann J, Sangha J, et al. The burden of cardiovascular disease amongst psychiatric patients. Int $\mathrm{J}$ Cardiol. 2013;169:e65-6.

2. Ames D, Camm J, Cook P, et al. Minimizing the risks associated with QTc prolongation in people with schizophrenia: a consensus statement by the Cardiac Safety in Schizophrenia Group. Encephale. 2002;28:552-62.

3. Larsen BA, Christenfeld NJS. Cardiovascular disease and psychiatric comorbidity: the potential role of perseverative cognition. Cardiovasc Psychiatry Neurol. 2009;2009:791017.

4. Bushe CJ, Taylor M, Haukka J. Mortality in schizophrenia: a measurable clinical endpoint. J Psychopharmacol. 2010;24:17-25.

5. Evans DL, Charney DS. Mood disorders and medical illness: a major public health problem. Biol Psychiatry. 2003;54:177-80.

6. De Hert M, Detraux J, van Winkel R, et al. Metabolic and cardiovascular adverse effects associated with antipsychotic drugs. Nat Rev Endocrinol. 2012;8:114-26.

7. Pacher P, Kecskemeti V. Cardiovascular side effects of new antidepressants and antipsychotics: new drugs, old concerns? Curr Pharm Des. 2004;10:2463-75.

8. Williams RB Jr, Haney TL, Lee KL, et al. Type A behavior, hostility, and coronary atherosclerosis. Psychosom Med. 1980;42:539-49.

9. Rugulies R. Depression as a predictor for coronary heart disease. a review and meta-analysis. Am J Prev Med. 2002;23:51-61.

10. Pae CU, Wang SM, Lee SJ, et al. Antidepressant and QT interval prolongation, how should we look at this issue? Focus on citalopram. Expert Opin Drug Saf. 2014;13:197-205.

11. Nachimuthu S, Assar MD, Schussler JM. Drug-induced QT interval prolongation: mechanisms and clinical management. Ther Adv Drug Saf. 2012;3:241-53.

12. FDA approves first drug to treat tardive dyskinesia [press release]. Silver Spring: US Food and Drug Administration; 2017. 
13. Cloud LJ, Zutshi D, Factor SA. Tardive dyskinesia: therapeutic options for an increasingly common disorder. Neurotherapeutics. 2014;11:166-76.

14. Zutshi D, Cloud LJ, Factor SA. Tardive syndromes are rarely reversible after discontinuing dopamine receptor blocking agents: experience from a university-based movement disorder clinic. Tremor Other Hyperkinet Mov (N Y). 2014;4:266.

15. Rana AQ, Chaudry ZM, Blanchet PJ. New and emerging treatments for symptomatic tardive dyskinesia. Drug Des Dev Ther. 2013;7:1329-40.

16. O'Brien CF, Jimenez R, Hauser RA, et al. NBI-98854, a selective monoamine transport inhibitor for the treatment of tardive dyskinesia: a randomized, double-blind, placebo-controlled study. Mov Disord. 2015;30:1681-7.

17. Hauser RA, Factor SA, Marder SR, et al. KINECT 3: a phase 3 randomized, double-blind, placebo-controlled trial of valbenazine for tardive dyskinesia. Am J Psychiatry. 2017;174:476-84.
18. Factor SA, Remington G, Comella CL, et al. The effects of valbenazine in participants with tardive dyskinesia: results of the 1-year KINECT 3 extension study. J Clin Psychiatry. 2017. https://doi.org/10.4088/JCP.17m11777 (Epub ahead of print).

19. Ingrezza [prescribing information]. San Diego: Neurocrine Biosciences, Inc.; 2017.

20. Jimenez R, Shiwach R, Bari M, O'Brien CF. 12-week treatment of tardive dyskinesia with NBI-98854 [poster no. 826]. Mov Disord. 2014;29(Suppl. 1):S304.

21. Combined list of drugs that prolong QT and/or cause torsades de pointes (TDP). Updated October 2017. http://crediblemeds.org/ pdftemp/pdf/CombinedList.pdf. Accessed 8 Nov 2017.

22. Milazzo V, Stefano CD, Servo S, et al. Drugs and orthostatic hypotension: evidence from literature. J Hypertens. 2012;1:104.

23. Xenazine [prescribing information]. Deerfield: Lundbeck; 2015. 